\title{
Embolisation procedures in congenital heart disease
}

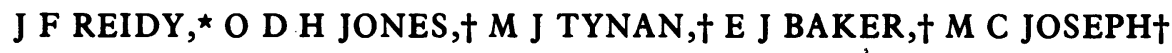 \\ From the ${ }^{\star D}$ epartments of Radiology and + Paediatric Cardiology, Guy's Hospital, London
}

SUMMARY Eight therapeutic embolisation procedures were performed by the transcutaneous catheter technique in seven patients with congenital heart disease. After surgical correction of tetralogy of Fallot (four patients), catheter embolisation was used to occlude two large aortopulmonary collaterals (one patient), three small aortopulmonary collaterals (one patient), and two Blalock-Taussig shunts (two patients). In two patients congenital coronary anomalies were occluded-a coronary arteriovenous malformation and a coronary artery/bronchial artery anastomosis. In one patient a pulmonary arteriovenous malformation was embolised.

Detachable balloons were used to occlude six large arteries, the three small arteries were occluded with small gelfoam fragments, and the pulmonary arteriovenous malformation was occluded with multiple steel coils and large gelfoam pieces. Successful occlusion was achieved in all cases. No complications were encountered and the procedure was well tolerated even in the two patients receiving postoperative intensive care.

Therapeutic embolisation in suitable cases is a safe and effective alternative to surgery and the detachable balloon technique is effective in occluding high flow vessels.

The scope and feasibility of interventional radiological techniques have increased dramatically in recent years. Although therapeutic embolisation procedures are now widely used in various clinical settings, their application to congenital heart disease has been limited.

Surgery remains the generally accepted treatment for significant arteriovenous fistulous connections related to the heart and great vessels. Access to these structures and their identification may be difficult, particularly when repeated operations are necessary. Moreover, it may be impossible to decide which structures may be ligated safely, for example when there is a dual blood supply to the lungs from both the pulmonary and systemic arterial systems. We describe here the application of embolisation techniques to various types of congenital heart disease.

\section{Patients and methods}

Between 1981 and 1984 therapeutic embolisation procedures were performed in six patients aged 3-67 years at this hospital, and one patient aged 21 years at the Westminster Hospital (see Table).

Requests for reprints to Dr J F Reidy, Department of Radiology, Guy's Hospital, London SE1 9RT.

Âccepted for publication 13 May 1985
Four patients with tetralogy of Fallot had undergone corrective surgery. They had all undergone surgical palliation earlier in life by insertion of BlalockTaussig shunts, in one case bilaterally. After corrective surgery, two had important residual aortopulmonary collaterals and two had patent Blalock-Taussig shunts. One of these Blalock-Taussig shunts had been ligated at corrective surgery (case 2).

Two patients had primary coronary vascular malformations-one had a very large circumflex artery to coronary sinus fistula associated with aneurysmal dilatation of the coronary sinus (case 7); the other had a large circumflex to bronchial artery anastomosis as well as a severe localised and proximal stenosis of the left anterior descending coronary artery (case 1).

One patient who had a large pulmonary arteriovenous malformation was cyanosed and polycythaemic (haemoglobin, $17 \mathrm{~g} / \mathrm{dl}$ ) (case 3). He had presented with a cerebellar infarct.

In all cases the patient's clinician had formed the view that treatment was indicated and that surgery should be undertaken if attempts at therapeutic embolisation failed. Informed consent to the embolisation procedure was obtained from either the patient or his parents.

The method of therapeutic embolisation depended on the size of the vessel to be occluded and the type of anomaly. The following methods were used for 
ible Embolisation procechures in patienss with congenital heart disease

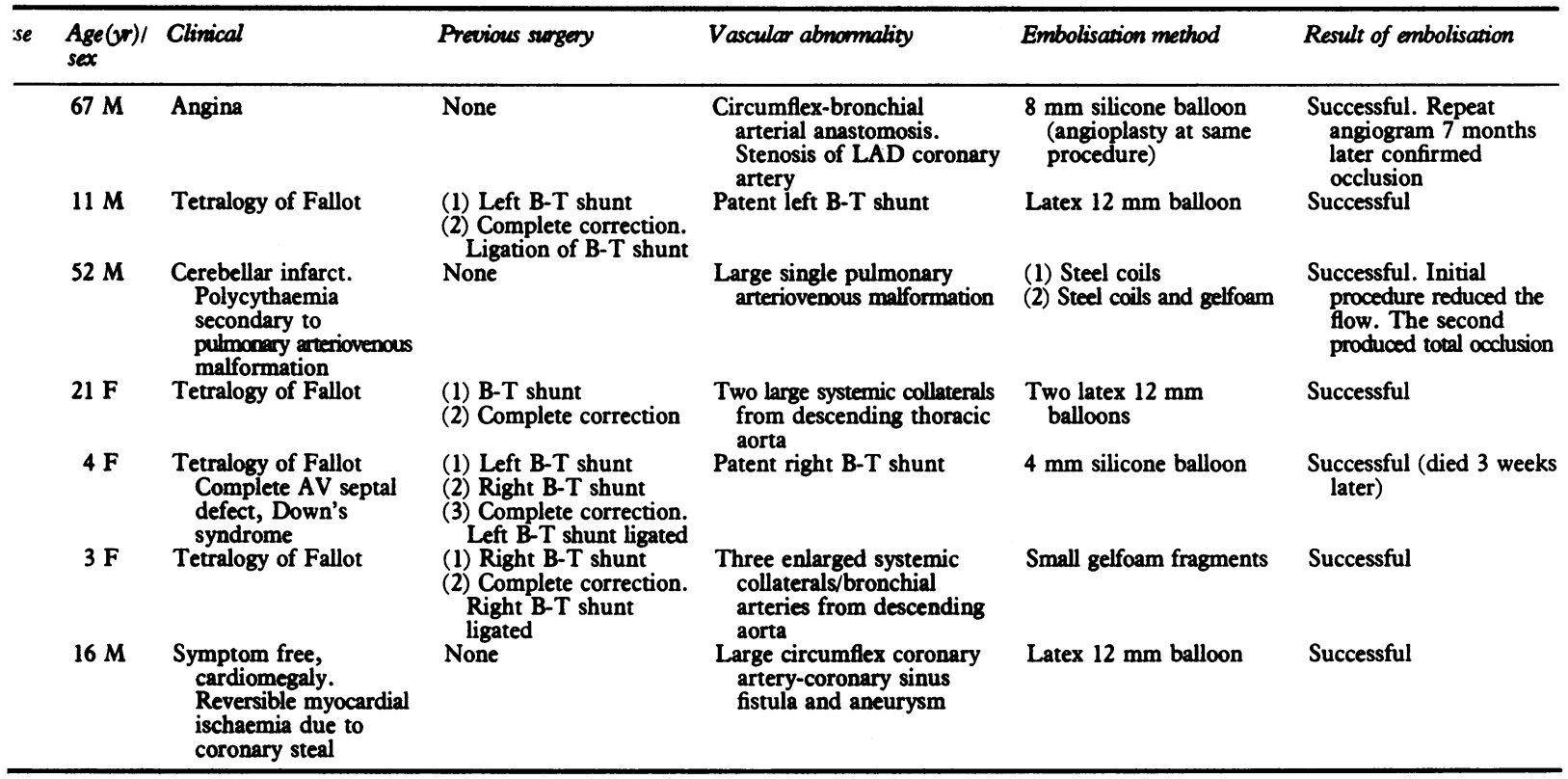

-T, Blalock-Taussig; AV, arteriovenous; LAD, left anterior descending.

embolisation: detachable balloons (five cases), silicone mini balloon (Becton-Dickinson) (cases 1 and 5), and latex balloons (Ingenor) (cases 2, 4, and 7); steel coils (William Cook Europe Aps) (case 3); and gelfoam (Sterispon, Allen and Hanburys) (cases 3 and 6).

Silicone mini balloons can be used to occlude vessels up to $8 \mathrm{~mm}$ in diameter. The smaller mini balloon ( $4 \mathrm{~mm}$ diameter) could be placed through a 5 French gauge catheter and this has advantages in small children. Where the vessels to be occluded were larger, latex balloons up to $12 \mathrm{~mm}$ in diameter were used. The silicone balloons are inflated with iso-osmotic contrast medium and have an extra advantage in that they may be fluid propelled. They are prepared ready for use, unlike the latex balloons which come in a kit form and have to be prepared and hand tied to the balloon catheter before use. The latex balloons are initially inflated with contrast medium to determine their position and their occluding effect. Contrast medium is then aspirated and replaced with an equal volume of a mixture of two silicone monomers. At body temperature these polymerise in about 15 minutes and then vulcanise. At this stage the process is no longer reversible. When the arteries to be occluded were small (case 6) and a detachable balloon or steel coil could not be safely inserted small particles of gelfoam (Sterispon) were injected through the catheter placed in the proximal vessel.

In the case of the pulmonary arteriovenous malfor- mation (case 3) in which it was considered appropriate to obliterate the malformation as well as the feeding arteries, multiple steel coils were used in addition to large gelfoam fragments. In this case two embolisation procedures were required to achieve complete occlusion.

\section{Results}

In all of five cases in which detachable balloons were used to occlude major arteries complete occlusion was confirmed by angiography at the end of the procedures (Figs. 1, 2, 3, and 4). In one of these patients (case 1) coronary arteriography seven months after the embolisation confirmed permanent occlusion as well as a good postangioplasty result (Fig. 1). In the other four cases follow up clinical evaluations, supported when appropriate by radionuclide studies, confirmed that successful occlusion had persisted.

In the patient (case 3) with the pulmonary arteriovenous malformation (Fig. 5a) the initial embolisation procedure with three large steel coils was only partly successful. Despite an improvement in the arterial oxygen saturation to within the normal range, a study with radionuclide labelled microspheres showed that right to left shunting though reduced was still appreciable. A pulmonary arteriogram three months after this embolisation procedure showed that the single feeding artery was still patent (Fig. 5b). 

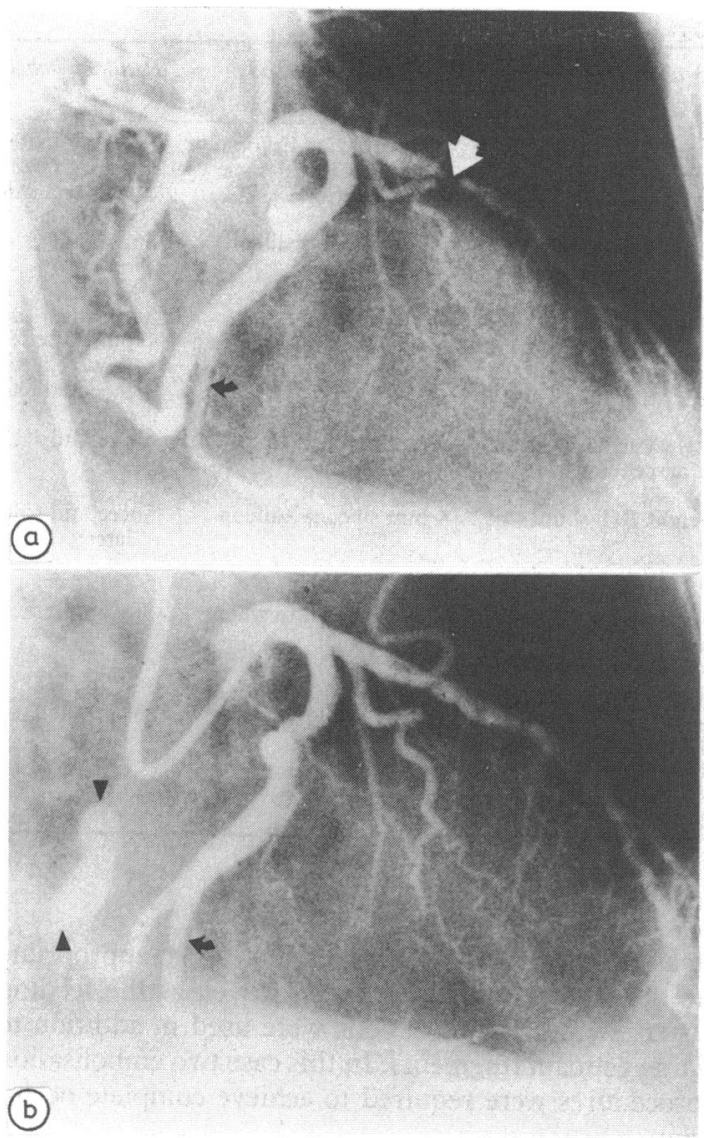

Fig. 1 (a) Left coronary arteriogram in case 1. Note the severe proximal stenosis of the anterior descending branch of the coronary artery (white arrowhead). The point of occlusion of the fistula must be distal to the last marginal branch (black arrow). (b) 7 months after embolisation and angioplasty. The balloon is filled with contrast (black arrowheads) and positioned well distal to the last marginal branch (black arrow). Good angioplasty result noted.

Complete occlusion was produced with three further steel coils and large gelfoam pieces (Fig. 5c). Subsequent radionuclide studies showed no evidence of right to left shunting. They also showed a segmental perfusion defect in the region of the occluded malformation.

The patient in whom multiple aortopulmonary collaterals were embolised with gelfoam particles showed clinical improvement, and successful occlusion of the arteries was seen angiographically (Fig. 6c). Radionuclide studies showed an appreciable decrease in the left to right shunt after the procedure.

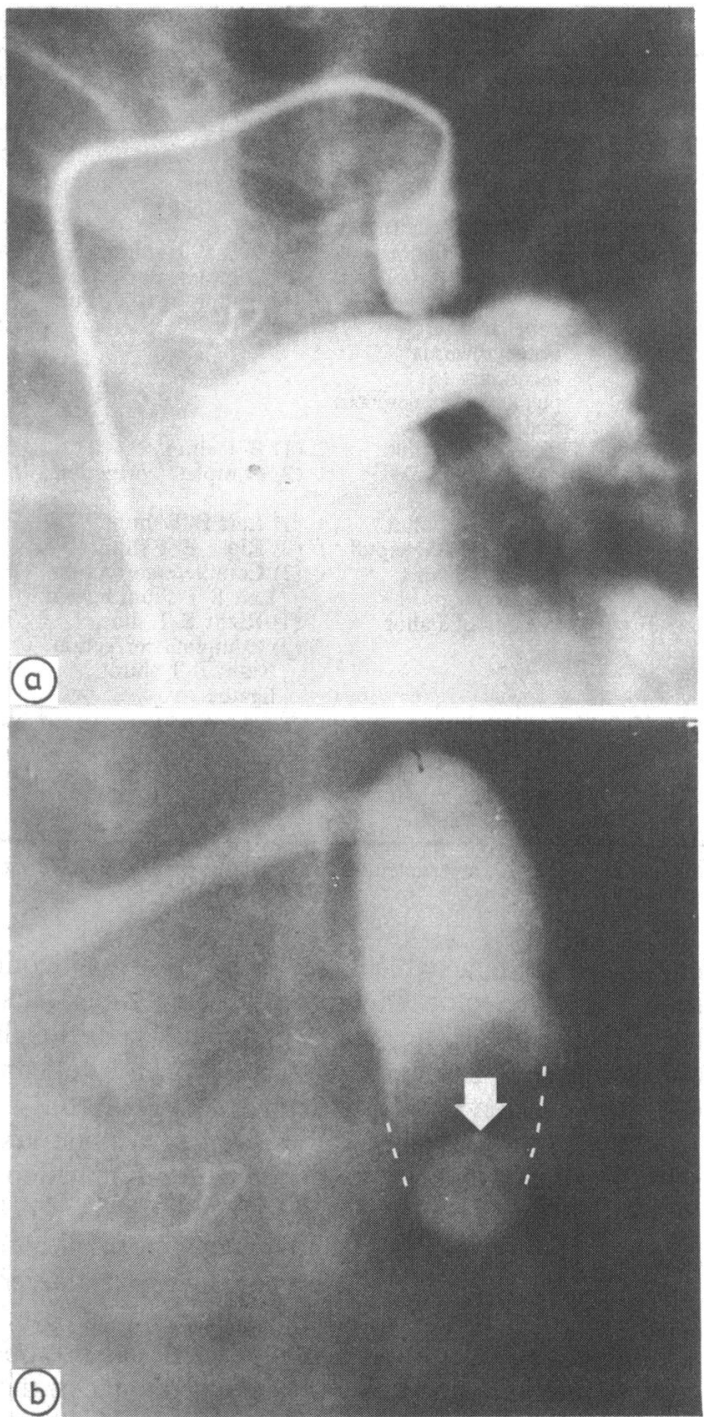

Fig. 2 (a) Injection into the Blalock-Taussig shunt (catheter via right sided aortic arch) in case 2 . Note the dilatation of the subclavian artery and the stenosis where it anastomoses with the left pulmonary artery. (b) Post embolisation injection of medivon into the Blalock-Taussig shumt shows occlusion and a radiolucent defect due to the silicone filled balloon. The rounded opacity is due to some residual contrast in the balloon, and the very small density area (white arrowhead) is a metal marker in the balloon.

In the patient with the left circumflex coronary artery-coronary sinus arteriovenous malformation (case 7) the flow through the circumflex coronary artery was so great that normal myocardial branches were not visualised when the aortic root or main coronary artery were injected (Fig. 4a). Only when a non-detachable balloon catheter with proximal injec- 

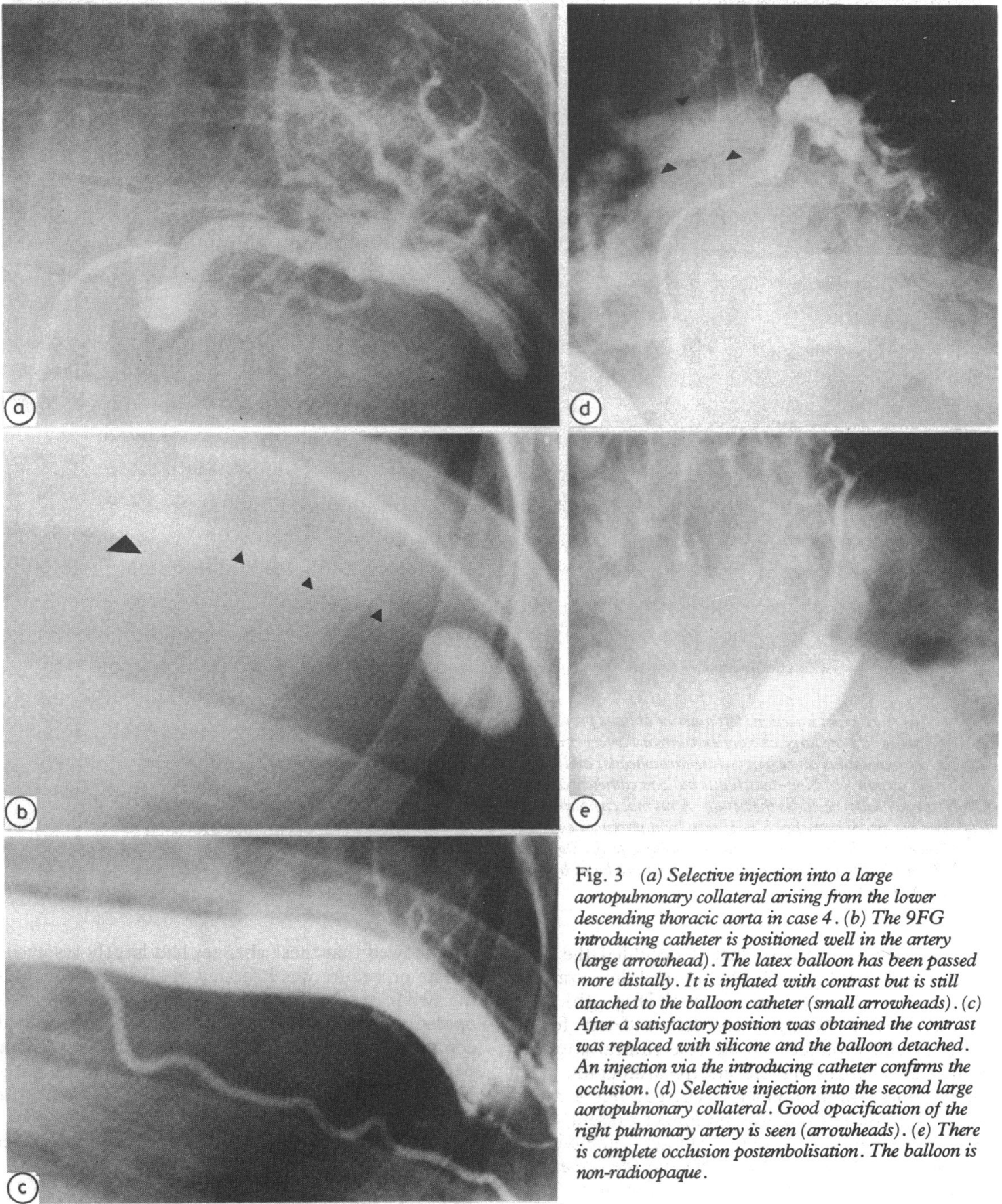

Fig. 3 (a) Selective injection into a large aortopulmonary collateral arising from the lower descending thracic aorta in case 4. (b) The 9FG introducing catheter is positioned well in the artery (large arrowhead). The latex balloon has been passed more distally. It is inflated with contrast but is still attached to the balloon catheter (small arrowheads). (c) After a satisfactory position was obtained the contrast was replaced with silicone and the balloon detached. An injection via the introducing catheter conforms the occlusion. (d) Selective injection into the second large aortopulmonary collateral. Good opacification of the right pulmonary artery is seen (arrowheads). (e) There is complete occlusion postembolisation. The balloon is non-radioopaque. 


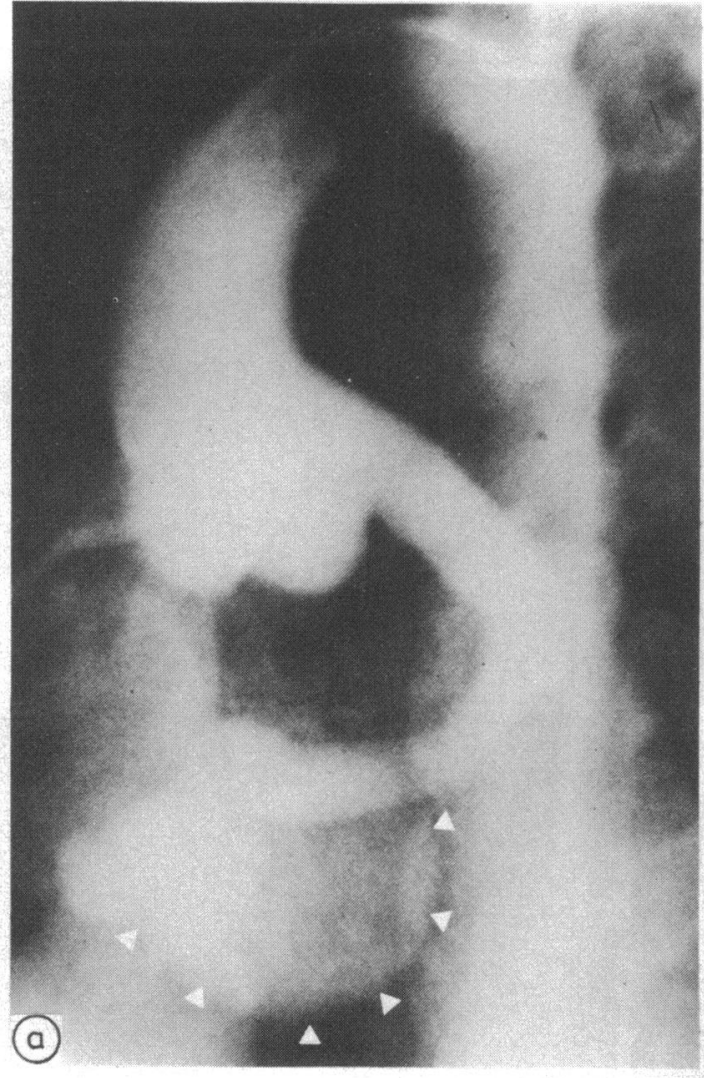

Fig. 4 (a) Aortic root injection (left anterior oblique projection) in case 7 shows a very large circumflex coronary artery draining into the coronary sirus anewrysm (white arrowheads) and thence to the right atrium. (b) Non-detachable balloon catheter (contrast filled) (arrowheads) occludes the fistula. A normal complement of left coronary artery branches is now seen by a proximal injection (right anterior oblique). (c) Left anterior oblique projection after balloon embolisation. Note the rounded defect produced by the silicone filled balloon.

tion holes was placed so that it occluded the distal artery and fistula could the normal left coronary branches be clearly demonstrated (Fig. 4b). This non-detachable balloon was left inflated for 15 minutes and produced no adverse clinical effects or ischaemic changes on the electrocardiogram. Some right bundle branch block occurred, but a month after embolisation this was no longer present. Coronary angiography after balloon embolisation confirmed occlusion of the fistula and the presence of all the normal coronary branches (Fig. $4 \mathrm{c}$ ). A preembolisation thallium computed tomogram showed reversible myocardial ischaemia due to a left coronary steal, and a repeat study three months after embolisa-
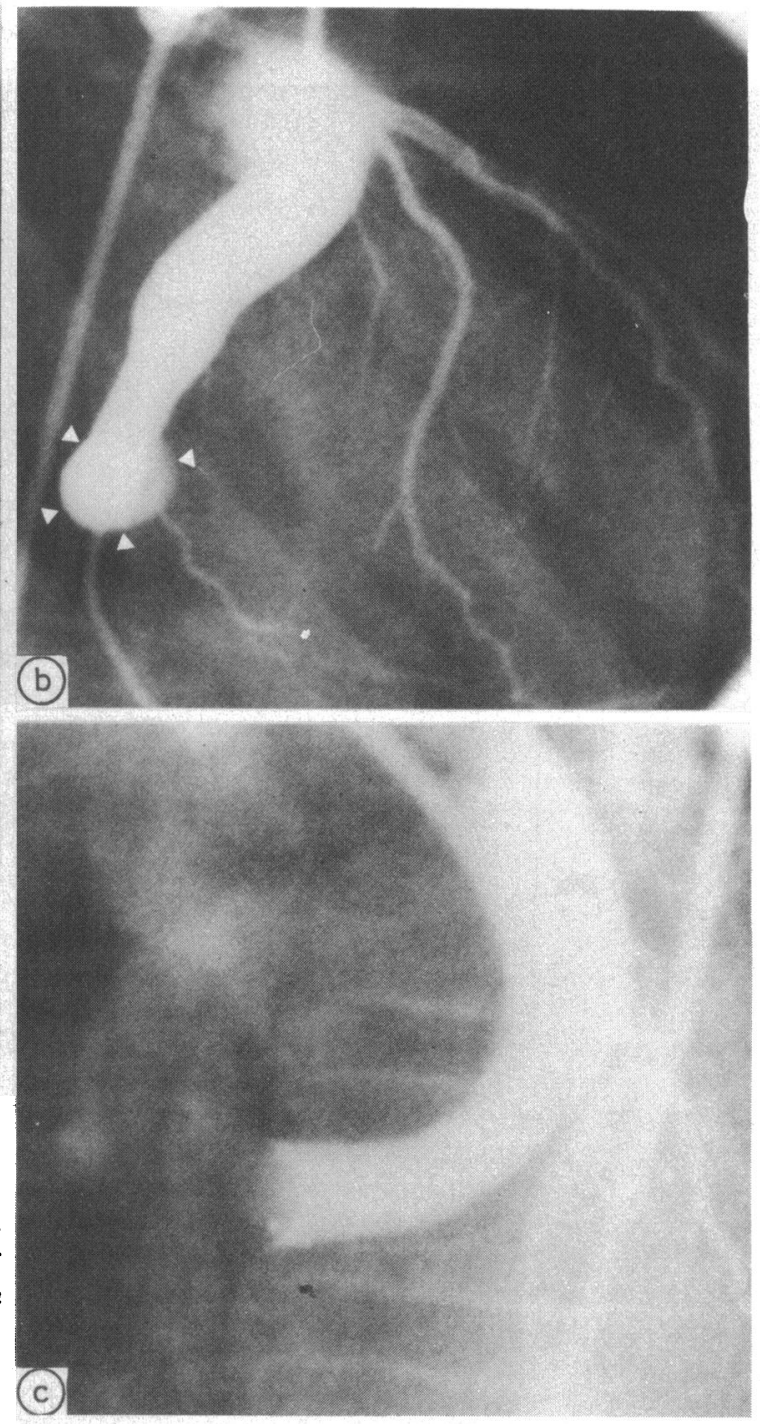

tion showed that these changes had largely resolved.

The procedure was tolerated in all patients including two (cases 5 and 6) who were still receiving postoperative intensive care. None of our patients had complications attributable to embolisation. One patient died (case 5) of a cause unrelated to embolisation three weeks after the procedure. To date, the remainder are alive and well.

Two patients (cases 1 and 2) have been the subject of previous case reports. ${ }^{12}$

\section{Discussion}

In recent years many methods of transcatheter embol- 

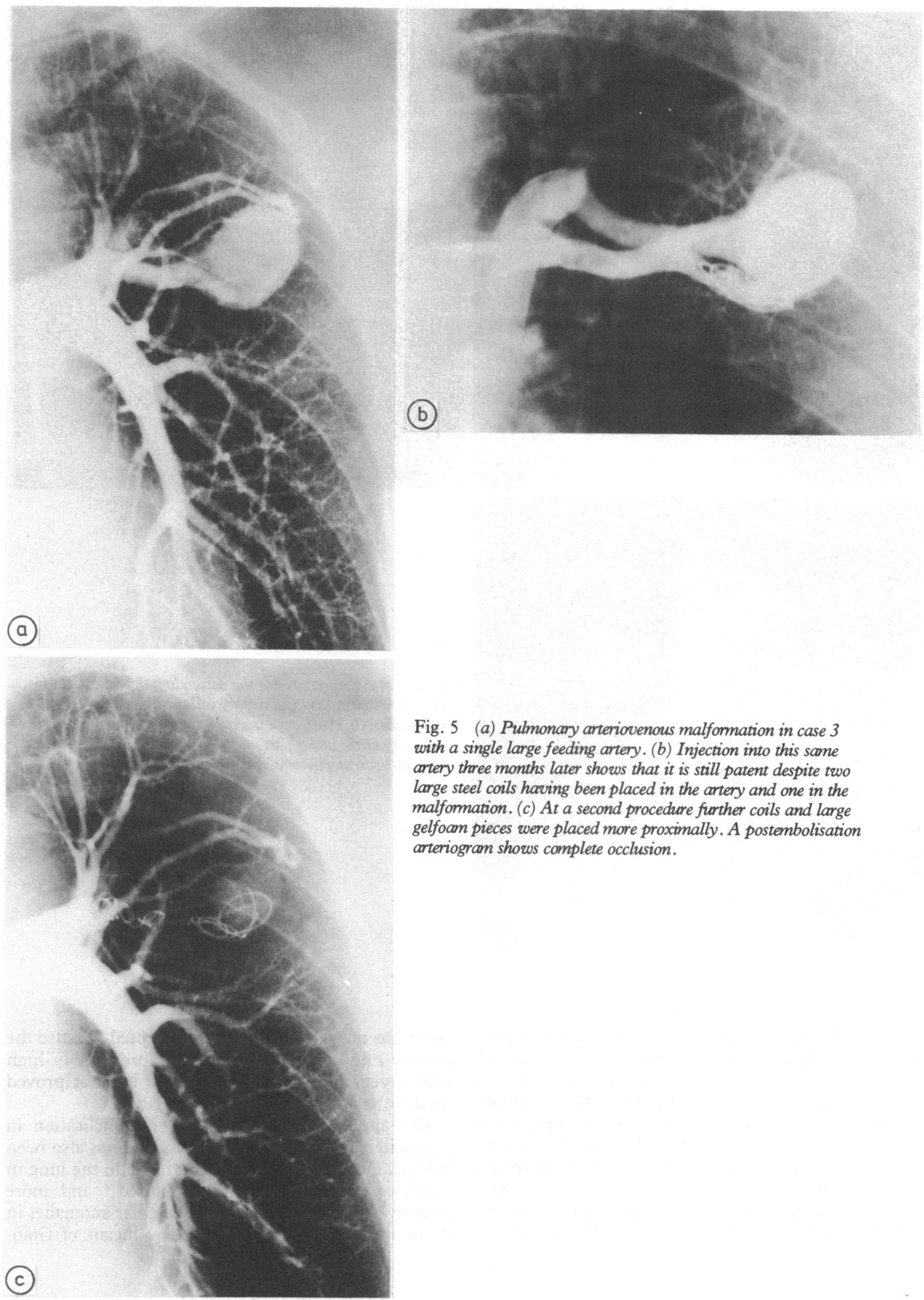

Fig. 5 (a) Pulmonary arteriovenous malformation in case 3 with a single large feeding artery. (b) Injection into this same artery three months later shows that it is still patent despite two large steel coils having been placed in the artery and one in the malformation. (c) At a second procedure further coils and large gelfoam pieces were placed more proximally. A postembolisation arteriogram shows complete occlusion. 

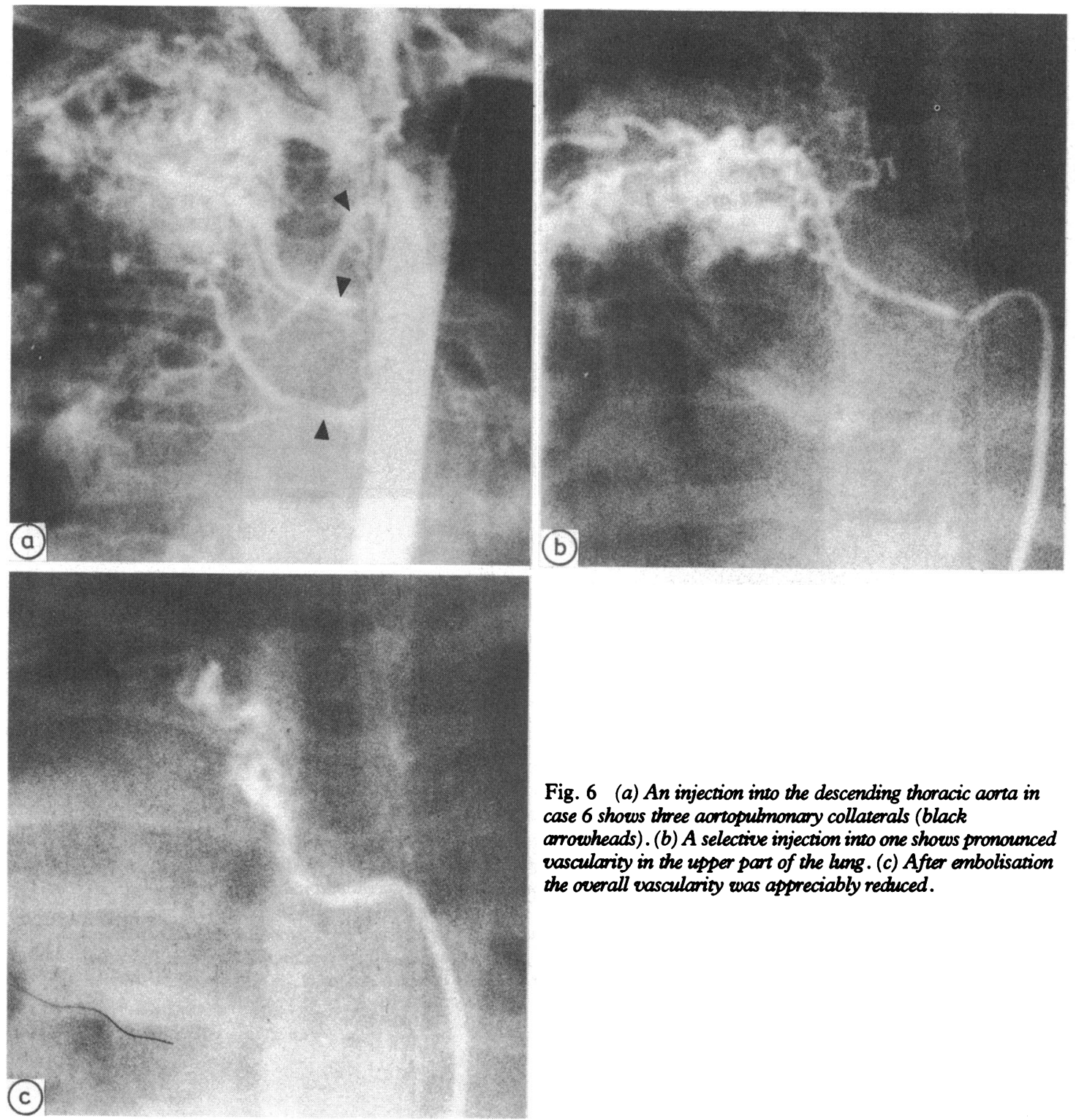

Fig. 6 (a) An injection into the descending thoracic aorta in case 6 shows three aortopulmonary collaterals (black arrowheads). (b) A selective injection into one shows pronounced vascularity in the upper part of the lung. (c) After embolisation the overall vascularity was appreciably reduced.

isation have been developed. Each has its advantages and disadvantages which must be taken into account when a decision is made on the type of procedure to use. Furthermore, the comparative risks of transcatheter embolisation and the surgical alternatives must be assessed since the clinical state of the patient and knowledge of the risks, failures, and complications of surgery will often dictate the course of action. Transcatheter occlusion of persistent ductus arteriosus was introduced more than 10 years ago, ${ }^{3}$ but it has not become generally accepted because the success rate of surgical closure is predictably high with a very low risk. Embolisation has not proved itself to be a simple and safe alternative.

The application of transcatheter embolisation in congenital cardiovascular malformation has also been limited. Occlusion of collateral arteries to the lung in tetralogy of Fallot has been reported, ${ }^{4}$ and more recently five assorted congenital vascular anomalies in the thorax have been embolised by means of Gian- 
turco steel coils. ${ }^{5}$ In one case the tissue adhesive Bucrylate was used to occlude a bronchial artery before surgical repair in tetralogy of Fallot. ${ }^{6}$ Apart from the embolisation of pulmonary arteriovenous malformations ${ }^{7}$ reports generally describe experience in single cases. ${ }^{8-10}$

Our experience with this varied group of cases prompts several comments. Firstly, in skilled hands these procedures are successful and we encountered no complications. Secondly, they can be carried out even in young children. Thirdly, embolisation can be successfully performed and is well tolerated in critically ill patients.

Detachable balloon embolisation was used in five of our seven cases. Balloon embolisation has been employed in only a very small fraction of all embolisation cases and has mainly been used in intracerebral vascular malformations. ${ }^{11}$ The major advantage of using detachable balloons is that they can be positioned in the vessel at the exact point where occlusion is required. Only when this position and the occluding effect have been confirmed by contrast medium injected through the introducing catheter is the balloon finally detached. Up to this time the process, unlike all other forms of embolisation, is reversible. In fistulous vascular connections and malformations with large vessels and high flow this avoids the risks of distal embolism. The high blood flow in large arteries may make them difficult to occlude completely. Detachable balloons of up to $12 \mathrm{~mm}$ in diameter can be used to occlude all except very large vessels. Several detachable balloon embolisation systems are available but only two were used in our patients. These balloons are biologically inert and produce permanent occlusion.

There are risks associated with the use of detachable balloons and operators must have experience in embolotherapy before attempting such procedures, especially in the heart. Difficulties with underinflation or use of too small a balloon may occur. Detachment of the balloon can then lead to pulmonary or, more seriously, systemic embolism. It may be difficult to detach silicone filled latex balloons. Alternatively premature detachment before vessel occlusion is a risk with the silicone balloons, especially in high flow arteries. Also early deflation can occur as they are filled with contrast media. Use of large introducing catheters and coaxial catheters in what may be lengthy procedures increases the risks of intravascular thromboembolic complications. These are reduced by routine heparinisation. ${ }^{12}$

Sometimes aortopulmonary collaterals consist of small and multiple arteries and thus are unsuitable for balloon embolisation. The precise site of embolisation in these end arteries, however, is not critical, and simpler embolisation materials can be used in such vessels. Radiologists have for many years been embolising hypertrophied bronchial and intercostal arteries in patients who have severe haemoptysis and chronic lung disease. Usually particulate emboli such as the biodegradable Sterispon have been used, although liquid embolic agents (alcohol, cyanoacrylate) have sometimes been advocated. Rarely a bronchial or intercostal artery also gives rise to a spinal medullary branch and spinal cord damage may be a complication of such embolisations. ${ }^{13}$ Before embolisation is considered, careful selective arteriograms (cut film is better than cine film) are essential. The presence of an anterior spinal artery arising from an intercostal/bronchial aortopulmonary collateral is an absolute contraindication to embolisation. With a careful approach and use of particulate rather than liquid emboli the risks appear to be very low. Of 104 patients who have had embolisation of bronchial and non-bronchial arteries for massive haemoptysis there were no medullary complications reported. ${ }^{14}$ Care must also be taken to avoid the more general complications of embolisation such as reflux of emboli and embolisation of distal aortic branches.

Multiple steel coils and large pieces of gelfoam were used to occlude the large pulmonary arteriovenous malformation. Though this was successful it took two procedures to occlude the vessel completely. Though others have used coils successfully to occlude pulmonary arteriovenous malformations, use of a single detachable balloon would probably have been as effective and perhaps more appropriate.

Only three of our seven patients had not previously had cardiac surgery. The other four cases all had complex congenital heart disease and had surgery on at least two occasions. In all four cases further surgery would have been necessary in the absence of an alternative embolisation technique. Two patients (cases 5 and 6) were regarded as poor surgical risks. In all our cases the aim of the embolisation was achieved and there were no complications related to the procedure.

Although the indications for these procedures are limited, there is scope for the extension of their use. For example, catheter embolisation of systemic to pulmonary collaterals in patients with multifocal blood supply can be performed. In the controlled environment of the catheter laboratory, trial reversible occlusions controlled by radioisotope imaging of the distribution of pulmonary perfusion ${ }^{15}$ could provide the ideal method of differentiating those collaterals which it is safe to occlude from those which it is not.

Our results strongly suggest that in skilled hands transcatheter embolisation is a safe and effective treatment of certain congenital cardiovascular malformations. Embolisation can be performed in critically ill children, and when vascular access is available 
it can be repeated when necessary. Embolisation procedures should be useful techniques complementary to surgery, and may in some instances obviate the need for operation.

We thank Dr J S Staffurth, Dr E Sowton, Mr P B Deverall, and Mr J C Lincoln for permission to report details of some of these cases.

\section{References}

1 Reidy JF, Sowton E, Ross DN. Transcatheter occlusion of coronary to bronchial anastomosis by detachable balloon combined with coronary angioplasty at same procedure. Br Heart F 1983; 49: 284-7.

2 Reidy JF, Baker E, Tynan M. Transcatheter occlusion of a Blalock-Taussig shunt with a detachable balloon in a child. Br Heart $\mathcal{F}$ 1983; 50: 101-3.

3 Porstmann W, Wierny L, Warnke H, Gertberger G, Romaniuk PA. Catheter closure of patent ductus arteriosus. Sixty-two cases treated without thoracotomy. Radiol Clin North Am 1971; 9: 203-18.

4 Yamamoto S, Nozawa T, Aizawa T, Honda M, Mohri $M$. Transcatheter embolization of bronchial collateral arteries prior to intracardiac operation for tetralogy of Fallot. F Thorac Cardiovasc Surg 1979; 78: 739-43.

5 Fuhrman BP, Bass JL, Castaneda-Zuniga W, Amplatz $\mathrm{K}$, Lock JE. Coil embolization of congenital thoracic vascular anomalies in infants and children. Circulation 1984; 70: 285-9.

6 Zuberbuhler JR, Dankner E, Zoltun R, Burkholder J,
Bahnson HT. Tissue adhesive closure of aorticpulmonary communications. Am Heart $\mathcal{F} 1974$; 88: 41-6.

7 Barth KH, White RI Jr, Kaufman SL, Terry PB, Roland JM. Embolotherapy of pulmonary arteriovenous malformations with detachable balloons. Radiology 1982; 142: 599-606.

8 Culham JAG, Izukawa T, Burns JE, Freedom RM. Embolization of a Blalock-Taussig shunt in a child. $A \mathcal{F R}$ 1981; 137: 413-5.

9 Grinnell VS, Mehringer CM, Hieshima GB, Stanley P, Lurie PR. Transaortic occlusion of collateral arteries to the lung by detachable balloons in a patient with tetralogy of Fallot. Circulation 1982; 65: 1276-8.

10 Florentine $M$, Wolfe RR, White RI. Balloon embolization to occlude a Blalock-Taussig shunt. I Am Coll Cardiol 1984; 3: 200-2.

11 Debrun G, Lacour P, Caron JP, Hurth M, Comoy J, Keravel Y. Detachable balloon and calibrated-leak balloon techniques in the treatment of cerebral vascular lesions. I Neurosurg 1978; 49: 635-49.

12 Debrun GM, Vinuela FV, Fox AJ. Aspirin and systemic heparinization in diagnostic and interventional neuroradiology. AFNR 1982; 3: 337-40.

13 Di Chiro G. Unintentional spinal cord arteriography: a warning. Radiology 1974; 112: 231-3.

14 Remy J, Arnaud A, Fardou H, Giraud R, Voisin C. Treatment of hemoptysis by embolization of bronchial arteries. Radiology 1977; 122: 33-7.

15 Baker EJ, Malamitsi J, Jones ODH, Maisey MN, Tynan MJ. Use of radionuclide labelled microspheres to show the distribution of the pulmonary perfusion with multifocal pulmonary blood supply. Br Heart f 1984; 52: $72-6$. 